\title{
Compact elliptical galaxies - compact bulges of stripped lenticulars/spirals?
}

\author{
Igor Chilingarian $^{1,2}$, Veronique Cayatte ${ }^{3}$, Laurent Chemin $^{4}$ and \\ Florence Durret ${ }^{5,1}$ \\ ${ }^{1}$ Observatoire de Paris, LERMA, UMR 8112, 61 Av. de l'Observatoire, Paris, 75014, France \\ email: Igor.Chilingarian@obspm.fr \\ ${ }^{2}$ Sternberg Astronomical Institute, Moscow State University, 13 Universitetski prospect, \\ 119992, Moscow, Russia \\ ${ }^{3}$ Observatoire de Paris, LUTH, UMR 8102, 5 pl. Jules Janssen, Meudon, 92195, France \\ ${ }^{4}$ Observatoire de Paris, GEPI, UMR 8111, 5 pl. Jules Janssen, Meudon, 92195, France \\ ${ }^{5}$ Institut d'Astrophysique de Paris, CNRS, UMR 7095, Université Pierre et Marie Curie, 98bis \\ Bd Arago, Paris, 75014, France
}

\begin{abstract}
We present discovery of compact elliptical galaxies in the centres of clusters and briefly discuss their evolution.
\end{abstract}

Keywords. galaxies: clusters: general, galaxies: bulges, galaxies: evolution

\section{Introduction}

Compact elliptical (cE) galaxies are extremely rare. Only five objects of this type have been identified so far: M32, NGC4486B, NGC5846A, two cEs in Abell 1689 (Mieske et al. 2005). These very unusual galaxies are thought to be generated by tidal stripping of intermediate-luminosity galaxies (see Choi et al. 2002 and references therein). Several observational projects aimed at searching for $\mathrm{cE}$ galaxies were conducted until now with no success (Drinkwater \& Gregg 1998, Ziegler \& Bender 1998).

\section{2. $\mathrm{cE}$ in Abell496}

We have discovered the sixth cE galaxy in the central region of the Abell 496 cluster, by analysing CFHT Megacam imagery, VLT-Giraffe multi-object spectroscopy, and archival HST WFPC2 data (Chilingarian et al. 2007). Velocity dispersion and stellar population (age, metallicity, $[\mathrm{Mg} / \mathrm{Fe}]$ ratio) of a newly discovered $\mathrm{cE}$ resemble properties of bulges of moderate-luminosity lenticulars, which is also the case for NGC4486B. Particularly small effective radius and high surface brightness put it on the continuation of the Kormendy (1977) relation for bulges and elliptical galaxies toward smaller spatial size, while it appears to be well above the majority of galaxies on the Faber-Jackson (1976) relation $(\sigma-L)$ due to its high velocity dispersion.

\section{Search for cE Galaxies using the Virtual Observatory}

We have conducted an automatic search for $\mathrm{cE}$ galaxy candidates in the nearby clusters using the power of the Virtual Observatory. We have developed a workflow - an automatic procedure for data discovery, retrieval, and analysis. It includes the following five steps: (1) querying NED to retrieve a list of Abell clusters having $z<0.05$; (2) querying HST 


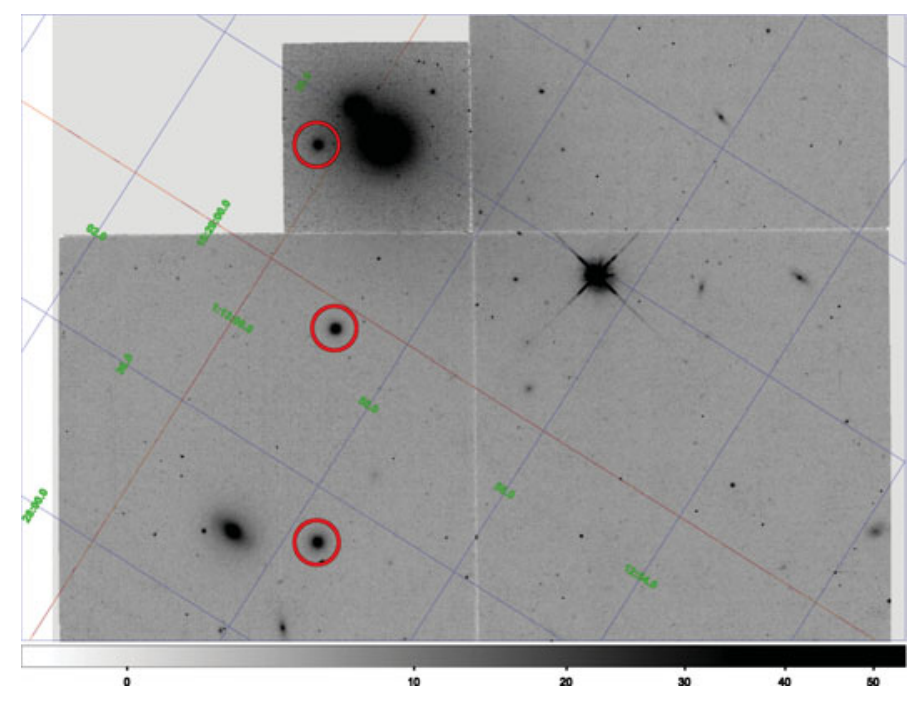

Figure 1. WFPC2 image of the central region of Abell 160. cE candidates are outlined.

WFPC2 and ACS associations (fully-reduced direct images) using IVOA Simple Image Access Protocol (SIAP); (3) running SExtractor as a remote tool on these images (no image download is required); (4) selecting extended objects having effective radii below $0.7 \mathrm{kpc}$ and B-band mean effective surface brightness higher than $20 \mathrm{mag} / \mathrm{arcsec}^{2}$; (5) querying NED to check if there are published redshifts for the selected objects. The light profiles of the candidate objects have been later analysed individually, resulting in a final sample of $12 \mathrm{cE}$ galaxies in 8 Abell clusters. Nearly all compact objects are located very close to $\mathrm{cD}$ galaxies $(d<40 \mathrm{kpc}$ in the projected distance). An example of the automatic cE candidate selection is shown in Fig. 1.

\section{Discussion}

We consider the scenario of tidal stripping of disc galaxies by the $\mathrm{cD}$ potential as a way to create cEs. Tidal stripping should remove 70 to 90 percent of the total mass, almost completely destroying the disky component, and significantly reducing bulge mass. This will reduce dynamical friction and allow galaxy to survive for a long time in the $\mathrm{cD}$ vicinity without being accreted by it. Since the stripping is done from the outer parts, the overall potential becomes more shallow and it may cause the bulge to shrink, leading to a compact object.

\section{Acknowledgements}

IC is grateful to the organizing committee of IAUS245 for provided financial support.

\section{References}

Chilingarian I., Cayatte V., Chemin L. et al. 2007, A\&A 466, L21

Choi P., Guhathakurta P., \& Johnston K. 2002, AJ 124, 310

Drinkwater M. J. \& Gregg M. D. 1998, MNRAS 296, L15

Faber S. M., \& Jackson R. E. 1976, ApJ 204, 668

Kormendy K. 1977, ApJ 218, 333

Mieske S., Infante L., Hilker M. et al. 2005, A $6 A$ 430, L25

Ziegler B. L. \& Bender R. 1998, A\&A 330, 819 